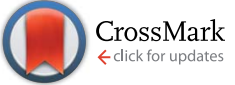

Cite this: RSC Adv., 2017, 7, 16104
Received 1st February 2017 Accepted 21st February 2017

DOI: $10.1039 / \mathrm{c} 7 \mathrm{ra01330b}$

rsc.li/rsc-advances

\title{
The enhancement of Hall mobility and conductivity of CVD graphene through radical doping and vacuum annealing $\dagger$
}

\begin{abstract}
Viet Phuong Pham, ${ }^{\text {*ab }}$ Anurag Mishra ${ }^{\text {ac }}$ and Geun Young Yeom*ab
We report an innovative method for chlorine doping of graphene utilizing an inductively coupled plasma system. TEM analysis reveals that the pre-doping (doping before wet transfer) and normal-doping (doping after wet transfer) were generally formed and trapped well between graphene layers; moreover, by thermal stability testing, the chlorine-trapped layer-by-layer graphene showed a very high thermal stability in vacuum at $230{ }^{\circ} \mathrm{C}$ for 100 hours. We also obtained the sheet resistance and optical transmittance of the $\mathrm{Cl}$-trapped tri-layer graphene at $72 \Omega \mathrm{sq}^{-1}$ and $95.64 \%$ at $550 \mathrm{~nm}$ wavelength, respectively. In addition, the high hole mobilities for the chlorine-trapped bi- and tri-layer graphene were observed up to 3352 and $3970 \mathrm{~cm}^{2} \mathrm{~V}^{-1} \mathrm{~s}^{-1}$, respectively.
\end{abstract}

\section{Introduction}

Graphene has attracted significant interest due to its exotic characteristics such as high mobility, high transmittance (\% T), and excellent thermal and electrical conductance. ${ }^{1-3}$ However, for many applications, graphene in its pristine form cannot be used due to the absence of a band-gap and high sheet resistance $\left(R_{\mathrm{S}}\right){ }^{4}$ Graphene synthesized using thermal chemical vapor deposition (CVD) and other methods show high $R_{\mathrm{s}}$ and low conductivity. Therefore, $R_{\mathrm{S}}$ reduction and conductivity enhancement of graphene are exciting topics. ${ }^{5-8}$

Doping is one of the best methods for $R_{\mathrm{S}}$ reduction using chemical doping ${ }^{6,9,10}$ and plasma doping. ${ }^{11-13}$ In the case of chemical doping, a layer-by-layer doping of 3 layers thin graphene films with $\mathrm{AuCl}_{3}$ yields a significant reduction in $R_{\mathrm{S}}$; however, it shows a sacrifice in the \% $T$ of the 3-layer doped graphene assembly (85\%). ${ }^{9}$ In addition, Tongay et al. presented p-type bis(trifluoromethanesulfonyl)amide doping of graphene with a significant reduction in the $R_{\mathrm{S}}$ value (425 to $129 \Omega \mathrm{sq}^{-1}$ ) and $\% T$ of $\sim 87 \% .{ }^{14}$ However, the limitation of chemical doping is that it has no long stability and the remaining chemical residues induce a deterioration in device performance. ${ }^{6}$

To date, there are no methods for the complete removal of chemical residues and these cannot be removed even via

\footnotetext{
${ }^{a}$ School of Advanced Materials Science and Engineering, Sungkyunkwan University (SKKU), Suwon, Gyeonggi-do 440-746, Republic of Korea. E-mail: pvphuong1985@ gmail.com; gyyeom@skku.edu

${ }^{b} S K K U$ Advanced Institute of Nano Technology (SAINT), SKKU, Suwon, Gyeonggi-do 440-746, Republic of Korea

${ }^{c}$ Etch Division, Applied Materials Co., 77, Sunnyvale, California, 94085, USA

$\dagger$ Electronic supplementary information (ESI) available. See DOI: 10.1039/c7ra01330b
}

chemical methods (acid), physical methods (Ar plasma cleaning) or both. ${ }^{\mathbf{1 4 - 2 6}}$ The existence of polymer residues or defects is inevitable because of the imperfect origin of CVD graphene. For instance, poly(methyl methacrylate) (PMMA) and contaminants can be attached at the edges of wrinkles, at the graphene domains, at grain boundaries, ${ }^{27}$ or bent graphene surfaces due to the highly rough $\mathrm{Cu}$ and imperfect processing during their synthesis. ${ }^{28}$

Plasma doping is an effective way to tune the graphene properties, as shown in a previous report. ${ }^{11}$ Chlorine $(\mathrm{Cl})$ plasma is the most controllable factor for graphene doping and can produce non-destructive doping with increased electrical conductivity. ${ }^{\mathbf{1 2}}$ The true potential of graphene lies in photonics and optoelectronics with exotic electronic and optical characteristics. This requires a very low $R_{\mathrm{S}}$ value for graphene with no degradation in transparency. Herein, we proposed a method based on an innovative inductively coupled plasma (ICP) system, which is of low energy and non-damaging ${ }^{29}$ for $\mathrm{Cl}$-doping in graphene with very low $R_{\mathrm{s}}$ values, extremely high transparency, high thermal stability, and high mobility.

\section{Results and discussion}

Monolayer graphene was grown on $\mathrm{Cu}$ via a CVD approach, as described in detail in a previous report. ${ }^{29}$ To optimize the Cldoping effect, pre-doping (doping before wet transfer) on graphene/Cu, normal-doping (doping after wet transfer), and a combination of pre-doping and normal-doping on the graphene/ substrate of PET and $\mathrm{SiO}_{2}$ were carried out. After Cl pre-doping, graphene/Cu was coated with PMMA, $\mathrm{Cu}$ was etched using a $\mathrm{FeCl}_{3}$ etchant over $45 \mathrm{~min}$, and then transferred on PET and $\mathrm{SiO}_{2}$. Finally, the PMMA was removed by washing the material with acetone, IPA, and DI water. For $\mathrm{Cl}$ normal-doping and 
combined-doping, which were applied for Cl-doped mono-, $\mathrm{Cl}$ trap-doped bi-, and tri-layer graphene, the doping process was repeated until 3 layers of graphene were achieved. Fig. 1(a) shows the strategy of Cl-trap-doping on layer-by-layer graphene utilized in this study. An innovative ICP source was used in the present study, with a dual mesh assembly inserted between the source and substrate as shown in Fig. 1(b). Fig. 1(c) depicts the crystal structure of the chlorinated graphene with the formation of covalent $\mathrm{C}-\mathrm{Cl}$ bonds on the CVD graphene surface. In this chlorine doping process, low energy radicals extracted from the chlorine plasma confined by a double-mesh grid system were used to prevent damage to graphene. When a $\mathrm{Cl}$ radical is adsorbed on the graphene surface, due to the strong electronegativity of $\mathrm{Cl}$ atom, an electron is transferred from the graphene surface to the attached $\mathrm{Cl}$ atom and an ionic bond between the graphene surface and $\mathrm{Cl}$ atom is formed without breaking the graphene structure. Generally, as previously reported, ${ }^{30}$ during plasma doping, graphene is damaged by breaking the $\mathrm{C}-\mathrm{C}$ bonds in graphene (the $\mathrm{C}-\mathrm{C}$ bond strength in graphene is very low at $4.9 \mathrm{eV}$ ) due to ion bombardment during exposure to the plasma.$^{30}$ Herein, in the $\mathrm{Cl}$ plasma, we used a double mesh-grid to remove the ion energy bombardment during the $\mathrm{Cl}$ plasma operation and to prevent the breaking of graphene network by the ions. Generally, chlorine dopants adsorbed on a graphene film surface can be easily removed during handling of the graphene under various environmental conditions such as heating, moisture, oxygen, etc. However, with covalent bonding, when the defect-containing fresh graphene on $\mathrm{Cu}$ foil is exposed to chlorine plasma, it is expected that chlorine can be bonded to the defect sites in the form of strong $\mathrm{C}-\mathrm{Cl}$ bonds (such as covalent bonding), which may remain even after the transfer process. In this case of covalent bonding, no increase in the number of defects (such as no increase of the D peak intensity in Raman spectroscopy) was observed because the $\mathrm{Cl}$ is bonded to pre-existing defects, as presented in Pham et al. ${ }^{29}$

To observe the low energy $\mathrm{Cl}$ adsorption on the graphene surface and interface, TEM analysis was utilized. Fig. 2(a) and (b) show the DF-TEM images of a bi-layer graphene without/ with $\mathrm{Cl}$ trap-doping. The white-colored clouds shown in Fig. 2(b) are believed to be the $\mathrm{Cl}$ atom locations caused by trap doping between the graphene layers. A thin network of white color is also shown in Fig. 2(a), which is believed to be the slight Cl-doping originating from the $\mathrm{FeCl}_{3}$ etchant. The existence of $\mathrm{Cl}$ atoms was also proven by micro-EDS in TEM with the analysis points confirming that the $\mathrm{Cl}$ adsorbates were small $(0.5 \%)$ and that they were sandwiched between two graphene layers, as shown in Fig. 2(c). In Fig. 2(c), the oxygen composition was increased from $0.96 \%$ (pristine bi-layer graphene) to $3.27 \%$ (Cltrapped bi-layer graphene) because all our experiments were carried out in an air atmosphere; in addition, the layer-by-layer graphene transfer was via wet method (PMMA). Therefore, reactions occur between the experimental processes with the contaminants, impurities in the air or the residues during wet transfer and these contaminants or residues contain oxygen functional groups $\left(\mathrm{OH}_{-}, \mathrm{COOH}-\right.$, etc. $)$, which are known as key factor in the deterioration of device performance. As the result, residues and impurities are inevitable during the CVD process of graphene, even a little bit. This is still a big challenge for 2Dmaterials research community. Recently, a promising method
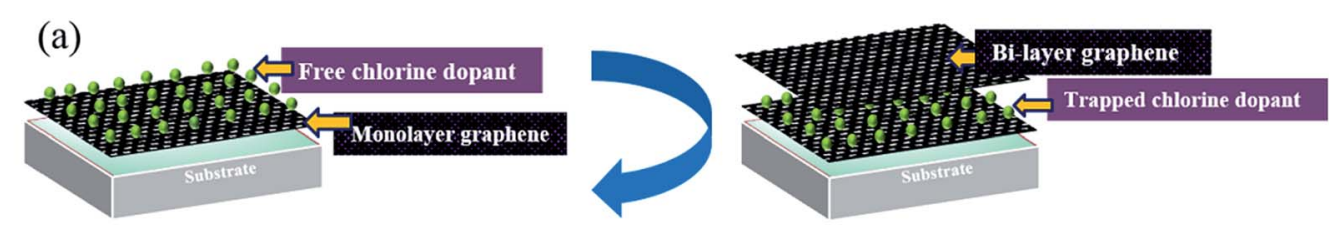

RF-ICP (13.56 MHz) System for Cl-Radical Doping

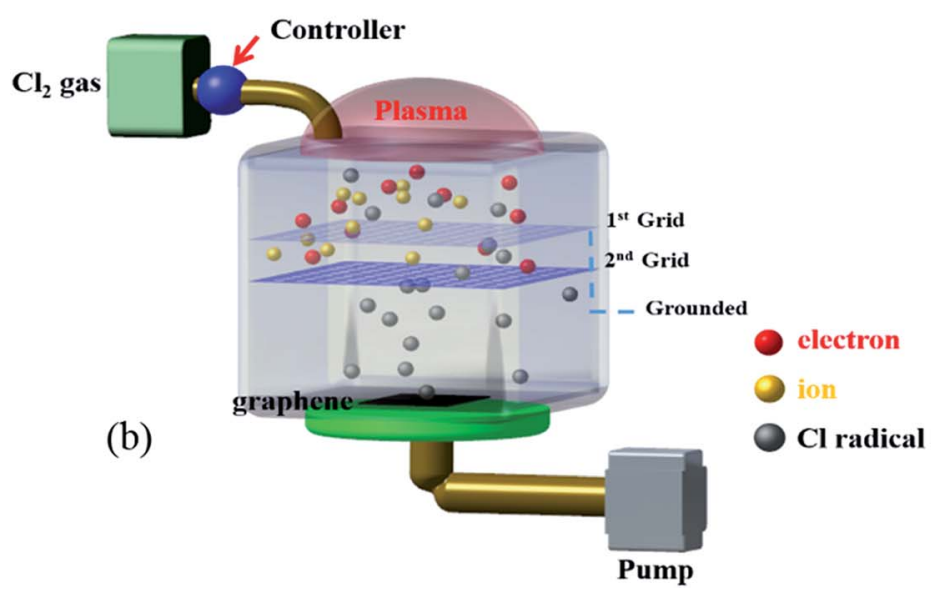

(c)



Fig. 1 (a) The strategy of Cl-trap doping on layer-by-layer graphene using the ICP system. (b) The low damage ICP source used in the present study, which is comprised of a dual mesh assembly inserted between the source and substrate. (c) An image of the crystal structure of chlorinated graphene with covalent $\mathrm{C}-\mathrm{Cl}$ bonding. 

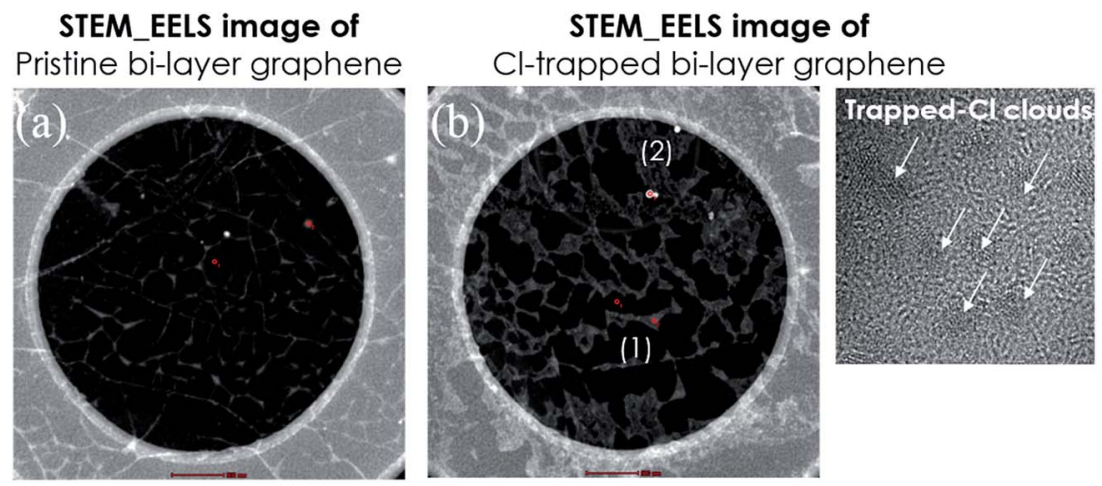

(c)

\begin{tabular}{c|c|c|}
\multirow{2}{*}{ Element } & \multicolumn{2}{|c|}{ Region } \\
\cline { 2 - 3 } & $\begin{array}{c}\text { Atomic \% } \\
(1)\end{array}$ & $\begin{array}{c}\text { Atomic \% } \\
(2)\end{array}$ \\
\hline $\mathrm{C}$ & 98.9 & 96.09 \\
\hline $\mathrm{O}$ & 0.96 & 3.27 \\
\hline $\mathrm{Cl}$ & 0.01 & 0.5 \\
\hline $\mathrm{Fe}$ & 0.13 & 0.14 \\
\hline Total & 100.00 & 100.00 \\
\hline
\end{tabular}

Fig. 2 TEM images ( $a$ and b) of pristine bi-layer graphene and the Cl-trapped bi-layer graphene. (c) Micro-EDS data of the atomic percentages in regions (1) and (2) of (b).

using the direct-growth of graphene on insulating substrates to avoid the wet transfer process was revealed and is still in progress. ${ }^{31}$

The Raman and XPS investigations used in this study have been investigated in detail in our previous report. ${ }^{29}$ In addition, we also showed the Raman spectra of tri-layer graphene with/ without p-type Cl-trap doping (see Fig. S1, ESI $\dagger$ ). With Cl-trap doping, the tri-layer graphene showed the blue-right shift in the $\mathrm{G}$ and $2 \mathrm{D}$ peaks (1577 and $2679 \mathrm{~cm}^{-1}$ ) when compared with the $\mathrm{G}$ and $2 \mathrm{D}$ peaks in the without Cl-trap doping of tri-layer graphene (1574 and $2675 \mathrm{~cm}^{-1}$ ), indicating the $\mathrm{Cl}$ dopant as a p-type dopant on graphene. In other words, the Raman spectra showed a blue-shift of $3 \mathrm{~cm}^{-1}$ and $4 \mathrm{~cm}^{-1}$ in the G and 2D peaks, indicating the $\mathrm{Cl}$ dopant as a p-type dopant on graphene. In fact, an investigation of the blue-shifts in the peaks of the Raman spectra of few-layer graphene has been carried out in detail in previous reports. ${ }^{9,32}$

For the long-term stability testing of $\mathrm{Cl}$ trap-doping on graphene, a thermal annealing process was carried out (Fig. 3). In fact, we kept some of the doped graphene samples used for the experiment, which were made four months earlier and, therefore, the $R_{\mathrm{s}}$ values were re-measured. We found no change in the $R_{\mathrm{S}}$ values after exposure to an air environment for four months. The $R_{\mathrm{S}}$ values for $130 \Omega \mathrm{sq}^{-1}$ of bi-layer graphene and $72 \Omega \mathrm{sq}^{-1}$ of tri-layer graphene were measured. Moreover, the four-monthold-doped graphene samples were heated for $100 \mathrm{~h}$ in a vacuum furnace at $230^{\circ} \mathrm{C}$ and, as shown in Fig. 3 , no significant change in the $R_{\mathrm{s}}$ values was observed. Therefore, it is believed that the doped grapheme samples prepared in our study are extremely stable.
Fig. 4(a) shows the Hall mobility values of the graphene samples without and with Cl-doping as a function of the $R_{\mathrm{s}}$ value. The Cl-doped mono-, bi-, tri-layer samples showed hole mobilities and $R_{\mathrm{s}}$ values of $\left(2750 \mathrm{~cm}^{2} \mathrm{~V}^{-1} \mathrm{~s}^{-1}, 305 \Omega \mathrm{sq}^{-1}\right),\left(3352 \mathrm{~cm}^{2} \mathrm{~V}^{-1}\right.$ $\left.\mathrm{s}^{-1}, 118 \Omega \mathrm{sq}^{-1}\right)$, and $\left(3970 \mathrm{~cm}^{2} \mathrm{~V}^{-1} \mathrm{~s}^{-1}, 72 \Omega \mathrm{sq}^{-1}\right)$, respectively. Due to the $\mathrm{Cl}$ p-type doping effect, these doped results are much higher when compared to those without Cl-doping.

The explanation of the mechanism of $\mathrm{Cl}$ p-type doping has been presented in detail in our previous report (with the strongly covalent $\mathrm{C}-\mathrm{Cl}$ bonds in the graphene lattice) ${ }^{29}$ Due to

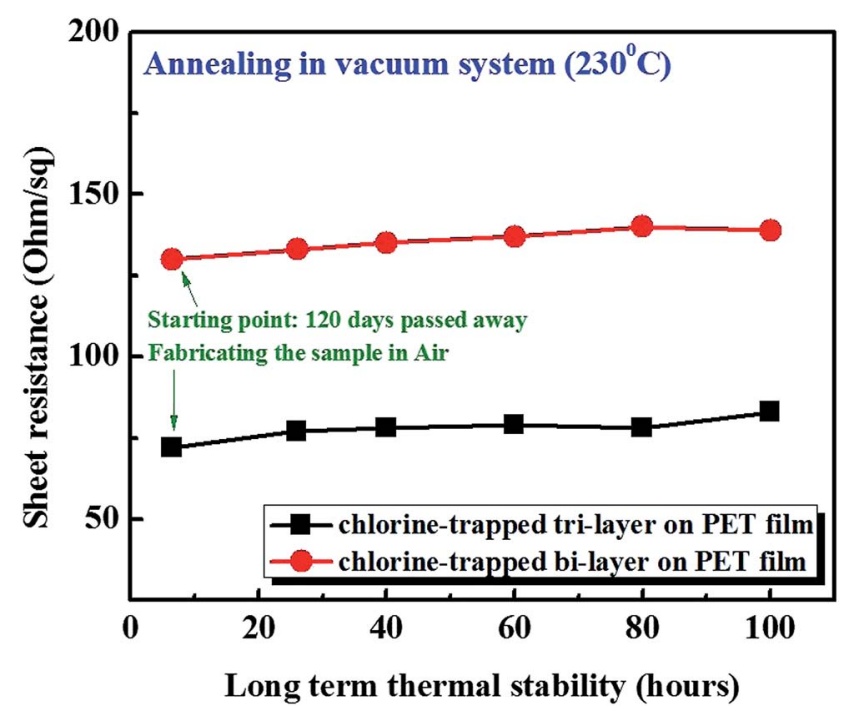

Fig. 3 Thermal stability testing of the Cl-trapped bi-layer and tri-layer graphene in a vacuum furnace. 

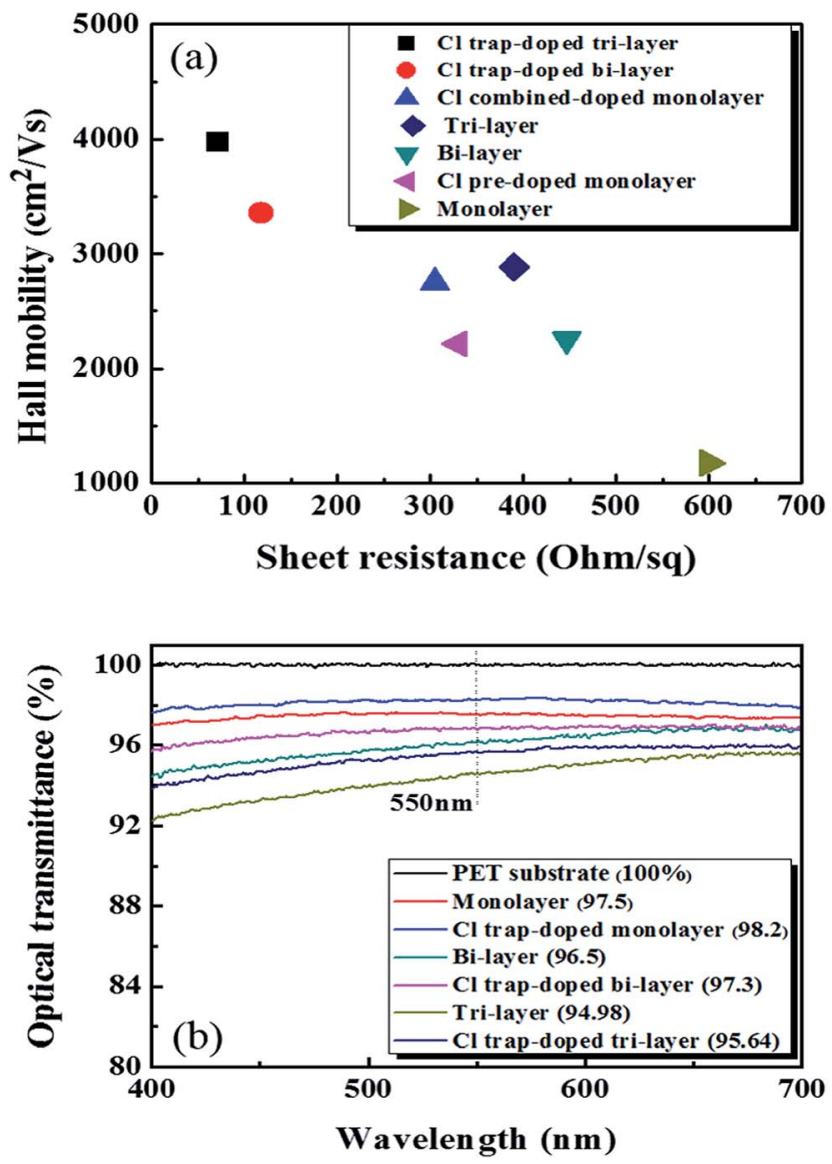

Fig. 4 (a) The Hall mobility values of graphene with/without Cl-doping as a function of the sheet resistance. (b) The optical transmittance of the graphene samples with/without $\mathrm{Cl}$-doping.

the Cl p-type doping, the $R_{\mathrm{S}}$ value was decreased and led to an enhancement in the conductivity and Hall mobility of graphene. The correlations between the $R_{\mathrm{S}}$ value, conductivity and mobility are depicted in the formulas: $R_{\mathrm{S}}=\rho L / A$ and $\rho=1 / \sigma=1 /$ $(e n \mu)$ where $\rho$ is the resistivity, $L$ is the length of the piece of graphene, $A$ is the cross-sectional area of the specimen, $\sigma$ is the conductivity, $e$ is the electron, $n$ is the carrier density, and $\mu$ is the mobility. In fact, these correlations have been welldescribed by De et al. ${ }^{33}$

The high \% $T$ of graphene layer and low $R_{\mathrm{S}}$ value are very important in transparent electrode-based applications. Pham et al. obtained the best values for $R_{\mathrm{s}}$ and \% $T$ in doped mono-layer graphene on a PET substrate at $240 \Omega \mathrm{sq}^{-1}$ and $97.7 \%$, respectively. ${ }^{29}$ Therefore, we measured the \% $T$ of the mono-, bi-, and trilayer graphene on PET doped with $\mathrm{Cl}$ plasma and the results are shown in Fig. 4(b). The monolayer graphene doped with the optimized doping process (90 s of pre-doping and $120 \mathrm{~s}$ of normal-doping) showed a high $\% T$ of $\sim 98.2 \%$ at $550 \mathrm{~nm}$. For the bi-layer graphene and tri-layer graphene doped via cyclic trap doping using Cl plasma, we measured the \% $T$ at 97.3 and $95.64 \%$ at $550 \mathrm{~nm}$, respectively. Because one monolayer graphene absorbs $\sim 2.3 \%$ at $550 \mathrm{~nm}$ (ref. 34) and the light excitation of $\mathrm{Cl}$ atoms, ${ }^{35-37}$ the $\% T$ of the doped mono- (98.2\%), bi- (97.3\%), and tri- $(95.64 \%)$ layer graphene samples that we observed in the experiments were a little higher than those of the undoped pristine mono- $(97.5 \%)$, bi- $(96.5 \%)$, and tri-layer graphene $(94.98 \%)$ samples.

\section{Conclusions}

In conclusion, we demonstrated the Cl-doping of graphene using an ICP system. Using TEM analysis, the Cl-trap doping strategy was generally formed well between the layer-by-layer graphene. Moreover, by thermal stability testing, the Cl-trapped layer-by-layer graphene showed a very high thermal stability in vacuum at $230{ }^{\circ} \mathrm{C}$ for 100 hours. We also obtained the $R_{\mathrm{s}}$ and \% $T$ of the Cl-trapped tri-layer graphene at $72 \Omega \mathrm{sq}^{-1}$ and $95.64 \%$ at $550 \mathrm{~nm}$, respectively. In addition, high hole mobilities of the Cl-trapped bi- and tri-layer graphene samples of up to 3352 and $3970 \mathrm{~cm}^{2} \mathrm{~V}^{-1} \mathrm{~s}^{-1}$, respectively, were achieved. This study opens the promising potential for utilizing a combination of $\mathrm{Cl}$ pre-doping and normal-doping for transparent graphene electrode-based applications, ${ }^{38}$ e.g. OLEDs, photodetectors, sensor, and solar cells.

\section{Experimental}

$\mathrm{Cu}$ foil with an area of $100 \times 90 \mathrm{~cm}^{2}$ and thickness of $75 \mu \mathrm{m}$ was rolled into a CVD vacuum chamber made of quartz. First, the discharge chamber was filled with $\mathrm{H}_{2}$ gas at the flow rate of 10 sccm and then the $\mathrm{Cu}$ foil was annealed for $1 \mathrm{~h}$ at $1050{ }^{\circ} \mathrm{C}$ under a $\mathrm{H}_{2}$ environment. Then, graphene was synthesized at $1050{ }^{\circ} \mathrm{C}$ under a $\mathrm{H}_{2} / \mathrm{CH}_{4}(10 / 20 \mathrm{sccm})$ environment for $30 \mathrm{~min}$ and then the chamber was cooled down to room temperature with $\mathrm{H}_{2}$ gas (10 sccm) for $1 \mathrm{~h}$. After the synthesis, the $\mathrm{Cu}$ foil was cut into small equal pieces $\left(3 \times 3 \mathrm{~cm}^{2}\right)$. These small pieces of graphene on $\mathrm{Cu}$ foil were affixed on glass substrates using tape and the glass substrate was used as a holder for the graphene-Cu foil assembly.

An innovative ICP source, with dual mesh assembly inserted between source and substrate to prevent the bombardment of energetic ions on the graphene surface, has been demonstrated in a previous report. ${ }^{29} \mathrm{The} \mathrm{Cl}_{2}$ plasma was generated under the conditions of $13.56 \mathrm{MHz}, 20 \mathrm{~W}, 10$ mTorr, and $60 \mathrm{sccm}$ for 90-120 s. The graphene films, which were inserted in the chamber, were cooled down to $15{ }^{\circ} \mathrm{C}$ using a chiller.

The $R_{\mathrm{S}}$ values of the graphene films on PET and $\mathrm{SiO}_{2}$ were measured utilizing an $R_{\mathrm{S}}$ meter (Dasoleng, FPP-2400). UV spectroscopy (Shimadzu, 3600) was used to measure the optical characteristics of the graphene films with/without $\mathrm{Cl}$ trapdoping. To observe the $\mathrm{Cl}$ atoms between the graphene layers, a micro-EDS (energy dispersive X-ray spectroscopy) installed in the DF (dark field)-TEM (Transmission Electron Microscopy, FEI Titan 80/300) and scanning transmission electron microscopyelectron energy loss spectroscopy (STEM-EELS) were utilized. The Hall carrier mobility was measured using a Hall effect measurement system (HMS-3000, ECOPIA). Raman spectroscopy (Renishaw, RM-1000 Invia) with an excitation energy of $2.41 \mathrm{eV}$ (514 $\mathrm{nm}, \mathrm{Ar}^{+}$ion laser) was used for the characterization of the graphene film trapped with $\mathrm{Cl}$ radicals. 


\section{Acknowledgements}

This research was supported by the Nano Material Technology Development Program through the National Research Foundation of Korea (NRF), funded by the Ministry of Education, Science and Technology (2016M3A7B4910429).

\section{Notes and references}

1 K. S. Novoselov, A. K. Geim, S. V. Morozov, D. Jiang, Y. Zhang, S. V. Dubonos, I. V. Grigorieva and A. Firsov, Science, 2004, 306, 666-669.

2 K. S. Novoselov, A. K. Geim, S. V. Morozov, D. Jiang, M. I. Katsnelson, I. V. Grigorieva, S. V. Dubonos and A. Firsov, Nature, 2005, 438, 197-200.

3 C. R. Dean, A. F. Young, C. Lee, L. Wang, S. Sorgenfrei and K. Watanabe, Nat. Nanotechnol., 2010, 5, 722-726.

4 D. W. Brenner, O. A. Shenderova, J. A. Harrison, J. S. Sttuart, B. Ni and S. B. Sinnott, J. Phys.: Condens. Matter, 2002, 14, 783-802.

5 C. Mattevi, H. W. Kim and M. Chhowalla, J. Mater. Chem., 2011, 21, 3324-3334.

6 H. Liu, Y. Liu and D. Zhu, J. Mater. Chem., 2011, 21, 33353345.

7 W. Chen, D. Qi, X. Gao and A. T. S. Wee, Prog. Surf. Sci., 2009, 84, 279-321.

8 S. M. Kim, A. Hsu, Y. H. Lee, M. Dresselhaus, T. Palacios, K. K. Kim and J. Kong, Nanotechnology, 2013, 24, 365602.

9 F. Gunes, H. J. Shin, C. Biswas, G. H. Han, E. S. Kim, S. J. Chae, J. Y. Choi and Y. H. Lee, ACS Nano, 2010, 4, 4595-4600.

10 J. Zheng, H. T. Liu, B. Wu, C. A. Di, Y. L. Guo, T. Wu, G. Yu, Y. Q. Liu and D. B. Zhu, Sci. Rep., 2012, 2, 662.

$11 \mathrm{X}$. Zhang, A. Hsu, H. Wang, Y. Song, J. Kong, M. S. Dresselhaus and T. Palacios, ACS Nano, 2013, 7, 7262-7270.

12 J. Wu, L. Xie, Y. G. Li, H. L. Wang, Y. Ouyang, J. Guo and H. Dai, J. Am. Chem. Soc., 2011, 13, 19668-19671.

13 B. Li, L. Zhou, D. Wu, H. Peng, K. Yan and Z. Liu, ACS Nano, 2011, 5, 5957-5961.

14 S. Tongay, K. Berke, M. Lemaitre, Z. Nasrollani, D. B. Tanner, A. F. Hebard and B. R. Appleton, Nanotechnology, 2011, 22, 425701.

15 N. Mcevoy, H. Nolan, N. A. Kumar, T. Hallam and G. S. Duesberg, Carbon, 2013, 54, 283-290.

16 N. Peltekis, S. Kumar, N. Mcevoy, K. Lee, A. Weidlich and G. S. Duesberg, Carbon, 2012, 50, 395-403.

17 P. Niranan, S. Marco, D. Alberto and A. Athanasia, J. Mater. Sci., 2011, 46, 5044-5049.
18 L. Xuelei, A. S. Brent, C. Irene, C. Guangjun, A. H. Christina, Z. Qin, O. Yaw, Y. Kai, P. Hailin, L. Qiliang, Z. Xiaoxiao, Z. Hui, R. H. W. Angela, L. Zhongfan, M. P. Lian and A. R. Curt, ACS Nano, 2011, 5, 9144-9153.

19 Y. F. Xiao, N. Ryo, C. Y. Li and T. Katsumi, Nanotechnology, 2010, 21, 475208.

20 C. Stephen, R. K. Peter and J. R. Preston, J. Vac. Sci. Technol., B: Microelectron. Nanometer Struct.-Process., Meas., Phenom., 1996, 14, 4129-4133.

21 A. M. Goosens, V. E. Calado, A. Barreiro, K. Watanabe, T. Taniguchi and L. M. K. Vandersypen, Appl. Phys. Lett., 2012, 100, 073110.

22 C. L. Yung, C. L. Chun, H. Y. Chao, J. Chuanhong, S. Kazu and W. C. Po, Nano Lett., 2012, 12, 414-419.

23 S. L. Wei, T. N. Chang and T. L. T. John, Nano Lett., 2014, 14, 3840-3847.

24 J. Moser, A. Barreiro and A. Batchtold, Appl. Phys. Lett., 2007, 91, 163513.

25 H. Micheal, B. Ryan and N. Lukas, Phys. Lett. A, 2013, 377, 1455-1458.

26 H. Terrones, R. Lv, M. Terrones and M. Dresselhaus, Rep. Prog. Phys., 2012, 75, 062501.

27 D. L. Duong, G. H. Han, S. M. Lee, F. Gunes, E. S. Kim, S. T. Kim, Q. H. Ta, K. P. So, S. J. Yoon, S. J. Chae, Y. W. Jo, M. H. Park, S. H. Chae, S. C. Lim, J. Y. Choi and Y. H. Lee, Nature, 2012, 490, 235-239.

28 G. H. Han, F. Gunes, J. J. Bae, E. S. Kim, S. J. Chae, H. J. Shin, J. Y. Choi, D. Pribat and Y. H. Lee, Nano Lett., 2011, 11, 41444148.

29 V. P. Pham, K. H. Kim, M. H. Jeon, S. H. Lee, K. N. Kim and G. Y. Yeom, Carbon, 2015, 95, 664-671.

30 D. W. Brenner, O. A. Shenderova, J. A. Harrison, J. S. Sttuart, B. Ni and S. B. Sinnott, J. Phys.: Condens. Matter, 2002, 14, 783.

31 H. Wang and G. Yu, Adv. Mater., 2016, 28, 4956-4975.

32 M. Chen, H. Zhou, C. Qiu, H. Yang, F. Yu and L. Sun, Nanotechnology, 2012, 23, 115706.

33 S. De and J. Coleman, ACS Nano, 2010, 4, 2713-2720.

34 R. R. Nair, P. Blake, A. N. Grigorenko, K. S. Novoselov, T. J. Booth, T. Stauber, N. M. R. Peres and A. K. Geim, Science, 2008, 320, 1308.

35 K. Suzuki, I. Nishiyama, Y. Ozaki and K. Kuchitsu, Chem. Phys. Lett., 1978, 58, 145-148.

36 J. B. Nee, Chin. J. Phys., 1988, 26, 254-261.

37 H. A. Bechtel, J. P. Canden, D. J. A. Brown, M. R. Martin, R. N. Zare and K. Vodopyanov, Angew. Chem., Int. Ed., 2005, 44, 2382-2385.

38 D. A. C. Brownson and C. Banks, Phys. Chem. Chem. Phys,, 2012, 14, 8264-8281. 